\title{
BETAXIN IN THE TREATMENT OF LEPROSY
}

\author{
Alfred Blueth.
}

A study of recent publications will confirm that very promising results are being obtained with " Betaxin " in many aetiologically diverse forms of polyneuritic and polyneuralgic disorder. The neurotoxic and infective types appear to respond equally well, and the striking successes obtained with this synthetic crystalline Vitamin- $B_{1}$ preparation suggest the presence of a $B_{1}$ deficiency in these conditions.

In view of these experiences Vitamin- $B_{1}$ was tried in the treatment of a very refractory infective type of peripheral neuritis, viz., leprous neuritis. The conditions underlying the use of Vitamin- $B_{1}$ in nervous forms of leprosy have been comprehensively laid down by Keil (I), and the service rendered by it in $\mathrm{I} 5$ cases are highly commended by Villela and Rocha (2). Their cases responded particularly well to intramuscular injections of " Betaxin," improvement being shown by a marked increase of appetite and weight and general betterment of health. The usual dosage was I $\mathrm{mg}$, and the total amount administered over some 9 weeks was $45 \mathrm{mg}$. Of late, in the light of further experience, these authors have been increasing the single dose up to ro $\mathrm{mg}$.

Badger and Patrick (3) also report favourable effects from intramuscular injections of a synthetic Vitamin- $B_{1}$ in 9 cases of nerve leprosy, particularly of the acute neuritic type. They also give about I mg. daily, and they state that the rapid cessation of pain and regression of the nerve swellings was most striking. Even advanced bilateral peroneus paralysis improved in a relatively short time so much that practically complete function could be restored.

Owing to the significance, both practical and theoretical, of these findings, some personal experiences of the writer may here be recorded :-

Case 1 Lepra mixta. Type $\mathrm{L}_{3} \mathrm{NI}$.

In April I938, a severe general reaction with intolerable pain, especially violent at night, a long the nerve trunks of the extremities. Neurotic vaso-dilatory reaction in hands and feet with well-marked areas of paraesthesia.

27.4.: Treatment begun with intramuscular "Betaxin" injections, the patient being given I mg. daily.

30.4.: The pains are less; patient sleeps better at night; hands and feet less swollen.

5.5.: No pain; swelling of hands and feet almost gone. 
II.5.: Still free from pain; swelling of hands and feet quite gone. Lepromata and other cutaneous manifestations of leprosy unaffected. "Betaxin " injections discontinued.

At the beginning of August, I938, another very violent general reaction with unendurable pain-this time, however, not of a neuralgic but of an arthritic character; all joints of the extremities very swollen. Since the usual treatment was without effect, "Betaxin" treatment was begun on 26.8. with daily injections of Io mg. No effect on the arthritic pains having been observed after ro injections, these were discontinued.

Case 2 Lepra mixta. Type L2NI.

Well-marked neurotic vaso-dilatory reaction of hands and feet accompanied by pain and paraesthesia formication and sensation of cold.

25.4.: Treatment begun with daily injections of "Betaxin" (dose: I mg. of synthetic Vitamin $B_{1}$ ).

27.4.: Swelling of hands and feet begin to go down. Paraesthesia improving.

30.4.: Continued improvement.

6.5.: Only the two forefingers remain somewhat livid and swollen.

II.5.: The swelling of the hands disappeared; the skin of the hands again displaying normal colour; pain and paraesthesia completely relieved. "Betaxin " treatment discontinued.

6.6.: Almost immediately after cessation of treatment the condition of the patient deteriorated and the former symptoms gradually returned.

I4.6.: Resumption of daily "Betaxin" injections (I mg. synthetic Vitamin- $B_{1}$ ).

23.6.: After Io injections, complete recovery. No further "Betaxin " treatment.

Up to the date of the last examination (20.ro.38) the patient's condition was entirely satisfactory and she has been able to resume her occupation as a sempstress.

Case 3 Lepra cutanea. Type L2.

In April, I938, simultaneously with a mild reaction of the skin symptoms, onset of violent pain in the nerves of the left arm.

Patient unable to sleep and much run down.

25.4.: Treatment begun with daily injections of "Betaxin" ( I mg. of synthetic Vitamin- $B_{1}$ ).

27.4.: Pain becoming less and patient sleeping better.

30.4.: Pain somewhat increased, especially at night. 
6.5.: Neuralgic pain now quite disappeared. No further injections.

At the time of the last examination (20.10.38) the patient was found to have remained free from symptoms.

Case 4 Lepra mixta. Type LIN2.

On 27th April, in the course of a severe general reaction, onset of extremely violent nerve pains in the extremities, especially in the legs. "Betaxin" (Io mg. of synthetic Vitamin- $B_{1}$ ) was injected.

28.4.: Pains considerably less. Injection repeated.

29.4.: Almost free from pain. Injection repeated.

30.4.: Injections discontinued; patient quite free from pain.

The general reaction remained unaffected but regressed slowly under the usual medication. The neuralgia had not reappeared up to the time of the last routine examination (20.10.38).

Case 5 Lepra mixta. Type $\mathrm{L}_{3} \mathrm{~N}$.

In June, 1938, during a severe reaction, patches of erythematous infiltration appeared over the whole body; livid swellings of the hands and feet; neuralgia and arthritic pains.

8.6.: Treatment begun with daily "Betaxin" injections ( $\mathrm{I} \mathrm{mg.}$ of synthetic Vitamin- $B_{1}$ ).

22.6.: Treatment discontinued. The neuralgia had completely ceased but the arthritic pains still persisted, although to a less extent. The regression of the neurotic vaso-dilatory symptoms in feet and hands was particularly rapid in this case. Other pathological changes in the skin, however, remained unaffected.

"Betaxin" injections were subsequently employed in all leprosy cases complicated by neuralgic symptoms and always with great success.

In conclusion it should be stated that treatment with "Betaxin" injections has proved of great service in leprous neuralgia occurring as an isolated symptom and in the course of a general reaction to the disease; the response is equally good in the usually intractable neurotic vaso-dilatory symptoms in the hands and feet, which as a rule are associated with severe pain and paraesthesia. The action appears to be highly selective, since the treatment leaves both the general and the arthritic symptoms of leprosy wholly unaffected.

\section{Literature.}

(I) Keil: " Is nerve leprosy aetiologically uniform? The Vitamin$\mathrm{B}_{1}$ treatment of nerve leprosy." Arch.f.Schiffs-und Tropenhyg, 1938, Vol. 42, No. I, pp.I-I2. 
(2) Villela and Rocha: "Influence of aneurine (Vitamin- $B_{1}$ ) and ascorbic acid (Vitamin-C) on leprosy.' Internat. Journ. of Leprosy, I938, Vol. 3, No. 3, p.458.

(3) Badger and Patrick: "Effect of intramuscular injections of Vitamin- $B_{1}$ on acute leprous neuritis and of oral administration on the general disease." Publ. Health Rep., Vol. 53, No. 24, I7.6.38, p.969. 